\title{
Cold Dark Matter Searches with the CDMS-II Experiment
}

\author{
Laura Baudis*t \\ University of Florida, USA \\ E-mail: lbaudiseufl.edu
}

The CDMS-II collaboration operates an experiment at the Soudan Mine, Minnesota to directly detect cold dark matter in the form of Weakly Interacting Massive Particles. The detectors are Ge (and Si) crystals of mass $250 \mathrm{~g}$ (100 g) cooled to $30 \mathrm{mK}$ and detect both the ionization and athermal phonon signals after a particle interacts in the Ge (Si) material. The athermal phonon readout utilizes superconducting $\mathrm{Al}$ films connected to tungsten transition edge sensors, while the charge is collected by applying a small (few V/cm) electric field across the crystals. The CDMS-II experiment has been operating $12 \mathrm{Ge}$ and $\mathrm{Si}$ detectors in their shielded environment at the Soudan Mine in 2004. No dark matter signal exceeding the expected background was found. The probed WIMP-nucleon cross section goes down to $1.6 \times 10^{-43} \mathrm{~cm}^{2}$ for a WIMP mass of $60 \mathrm{GeV} / \mathrm{c}^{2}$. I will present the apparatus, show the latest results for WIMP searches and discuss the current status and future plans.

International Europhysics Conference on High Energy Physics July 21st - 27th 2005

Lisboa, Portugal

\footnotetext{
*Speaker.

${ }^{\dagger}$ for the CDMS-II Collaboration
} 


\section{Introduction}

The gravitationally clustered matter in the Universe could be made of Weakly Interacting Massive Particles (WIMPs), which are thermal relics of the hot Big Bang. If such particles exist and have a relic density around $\Omega=0.2$ we expect their masses and annihilation cross sections to be at the weak scale. WIMPs can be detected on Earth via their elastic scattering with nuclei. The deposited energies are few tens of $\mathrm{keVs}$ and the expected event rates are well below 1 event $\mathrm{kg}^{-1} \mathrm{~d}^{-1}$. Although a vast effort is dedicated to search for dark matter particles, no unambiguous evidence has been delivered so far [1]. The world's most stringent limits on WIMP-nucleon cross sections come from the CDMS-II experiment. In the following, I will briefly describe the CDMS apparatus, show the latest WIMP search results obtained by operating $12 \mathrm{Ge}$ and $\mathrm{Si}$ detectors at Soudan in 2004, discuss the status of the current run with 30 detectors and present our future plans to explore the parameter space predicted for WIMP masses and cross sections.

\section{Experimental Apparatus and Recent Results}

The experiment is operated at a depth of 2080 mwe in the Soudan Mine, where the muon flux is reduced by a factor of $10^{4}$ compared to the Earth's surface. It is further shielded against environmental radioactivity and muon induced backgrounds by lead and polyethylene layers, and surrounded by an active plastic scintillator veto system. The actual detectors are located inside a concentric set of $\mathrm{Cu}$ cans (icebox) coupled to the different thermal stages of a dilution refrigerator. CDMS uses advanced Z(depth)-sensitive Ionization- and Phonon (ZIP) detectors, which simultaneously measure the ionization and athermal phonon signals after a particle interacts in the crystal. The athermal phonons are detected using quasiparticle-trap-assisted electrothermalfeedback transition-edge-sensors [2]. They consist of photolitographically patterned, overlapping thin films of superconducting aluminum and tungsten, divided into 4 independent channels. The sensors are intrinsically very fast, with $\sim 100 \mathrm{~ns}$ rise times and $20-40 \mu$ s fall times. The actual ZIP pulses have rise times of 5-15 $\mu \mathrm{s}$ and fall times of $\sim 100 \mu \mathrm{s}$, these being dependent on the phonon propagation in the crystal and the quasiparticle diffusion in the aluminum fins. The pulse rise times are sensitive to the phonon arrival times at each of the four quadrants and allow to localize an event in the $\mathrm{x}-\mathrm{y}$ plane with $1 \mathrm{~mm}$ precision. To perform the ionization measurement, a drift field of a few $\mathrm{V} / \mathrm{cm}$ is applied across the crystal using electrodes deposited on the two faces of each detector. The electrodes are segmented into an outer guard ring and a central disk-shaped volume, allowing to veto events near the edge of the crystals. A stack of six Ge or Si detectors together with the corresponding cold electronics is named a 'tower'. More details on CDMS-II can be found in [3].

The ZIP detectors provide event-by-event discrimination of nuclear recoils from the dominant background of electron recoils. The ratio of ionization energy to phonon energy (ionization yield) is $\sim 0.3$ for nuclear recoils in $\mathrm{Ge}$, and $\sim 0.25$ in $\mathrm{Si}$. The ionization yield is $\sim 1$ for electron recoils with full charge collection. Electron recoils near the detector surface suffer from poor ionization collection and can mimic a nuclear recoil. The timing properties of the 4 phonon signals yield a powerful method to discriminate against such surface events [7] (as shown in Fig. 1, left).

In 2004, two towers were operated for 74.5 live days at Soudan, yielding an exposure of $34 \mathrm{~kg} \mathrm{~d}$ in $\mathrm{Ge}$ and $12 \mathrm{~kg} \mathrm{~d}$ in $\mathrm{Si}$ in the $10-100 \mathrm{keV}$ nuclear recoil energy range. In order to avoid 

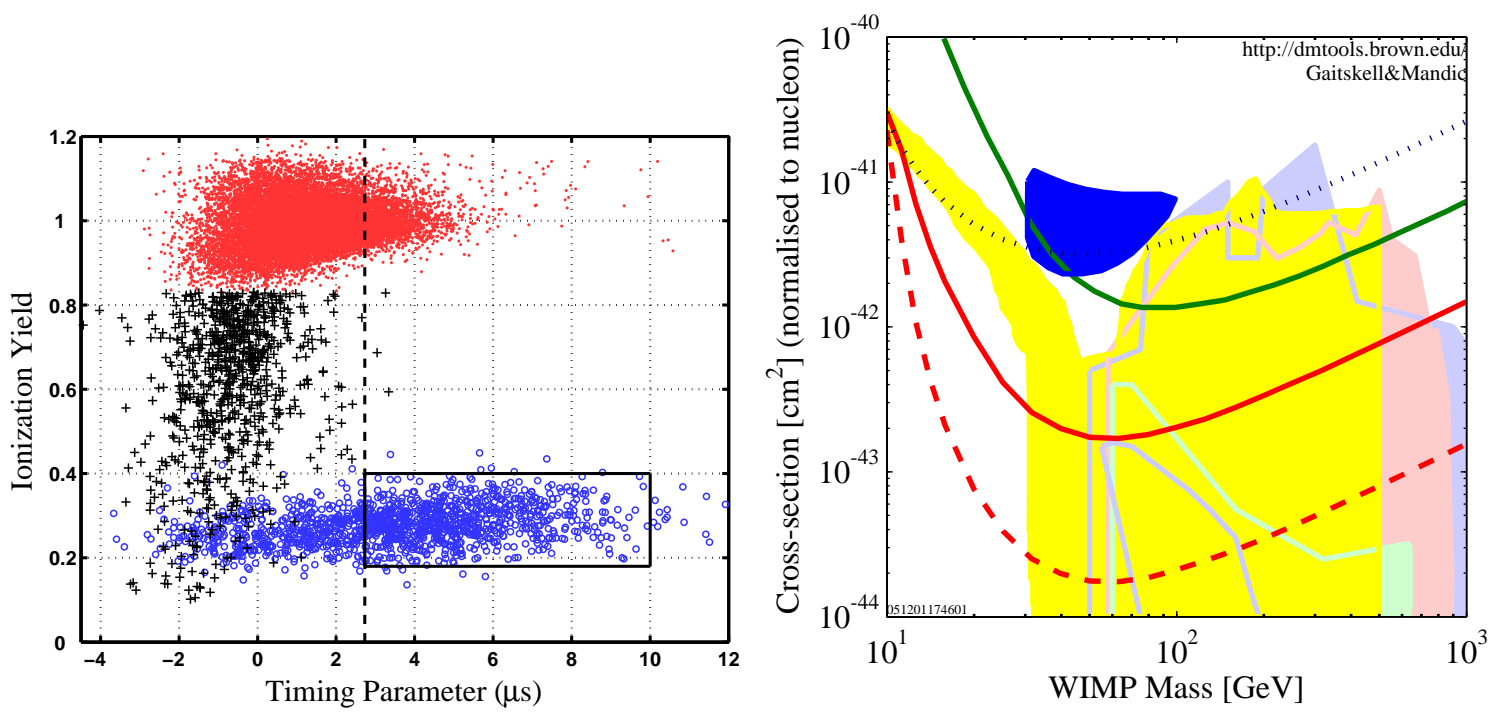

Figure 1: Left: Ionization yield versus phonon timing parameter for ${ }^{133} \mathrm{Ba}$ gamma calibration events (dots and crosses) and ${ }^{252} \mathrm{Cf}$ neutron calibration events (circles). Low-yield ${ }^{133} \mathrm{Ba}$ events (crosses) have small values of the timing parameter, a typical cut is indicated by the dashed vertical line. Right: Experimental results and theoretical predictions for spin-independent WIMP nucleon cross sections versus WIMP mass. The data (from high to low cross sections) show the DAMA allowed region (blue) [4], the recent EDELWEISS result (green) [5] and the CDMS Si (blue dotted) and Ge (solid red) results [7]. Also shown is the expectation for 5 CDMS towers at Soudan (red dashed). The SUSY theory regions are taken from [6].

bias, the analysis was performed 'blind', with events in and near the signal region removed from the WIMP-search data ('masked'). The cuts defining a signal were determined using calibration data from ${ }^{252} \mathrm{Cf}$ and ${ }^{133} \mathrm{Ba}$ sources, as well as non-masked WIMP-search data. After un-masking the data, one candidate nuclear recoil event at $10.5 \mathrm{keV}$ was observed in $\mathrm{Ge}$, while no events were seen in the Si data [7]. Although the candidate event occurred in a Ge detector during a period of inefficient ionization collection, the result is consistent with the expected background from surface events. The new upper limit on spin-independent WIMP-nucleon cross sections is $1.6 \times 10^{-43} \mathrm{~cm}^{2}$ from $\mathrm{Ge}$, and $3.4 \times 10^{-42} \mathrm{~cm}^{2}$ from $\mathrm{Si}$, both at the $90 \% \mathrm{CL}$ and at a WIMP mass of $60 \mathrm{GeV} / \mathrm{c}^{2}$ [7] (see Fig. 1, right). The limits on spin-dependent WIMP interactions, shown in Fig. 2 (left), are competitive with other experiments, in spite of the low abundance of ${ }^{73} \mathrm{Ge}(7.8 \%)$ in natural germanium. In particular, in the case of a pure neutron coupling, CDMS yields the most stringent limit obtained so far, thus strongly constraining interpretations of the DAMA signal region [8].

\section{Plans for the Future}

After the end of the two-tower run, the Soudan cryostat was warmed up and three additional towers were installed (see Fig. 2, right). Although the five towers were successfully cooled down to $40 \mathrm{mK}$ in July 2005, the noise associated with a cryocooler installed to remove the extra heatload was too high for stable operation. A vibration isolation system has now been constructed, and will be mounted at Soudan before the end of 2005. It is expected that CDMS-II will start the five-tower run at the beginning of 2006. The predicted sensitivity with $4.5 \mathrm{~kg}$ of Ge and $1 \mathrm{~kg}$ of Si 

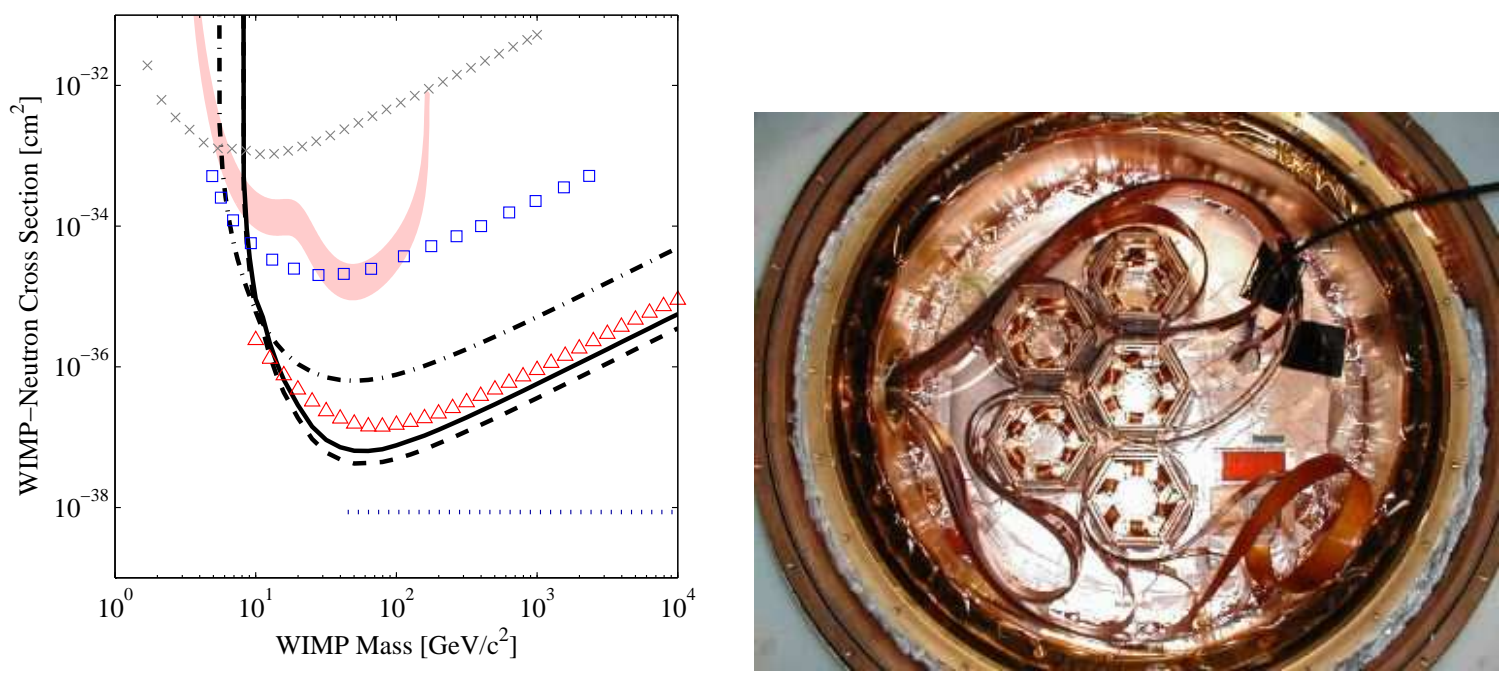

Figure 2: Left: Experimental results for spin-dependent WIMP couplings (90\% C.L. contours), for the case of a pure neutron coupling. The CDMS limits from Ge ( $\mathrm{Si}$ ) are shown in dark solid (dash-dot). Details can be found in [8]. Right: The five detector towers during their installation in the Soudan icebox. Visible is the top section of each hexagonal tower, with the cold electronics (FETs and SQUIDs) cards and the striplines. Six ZIP detectors (not visible) are stacked below each tower.

is shown in Fig. 1. To considerably extend the reach in the predicted WIMP parameter space, the CDMS collaboration has proposed the SuperCDMS program [9], which would utilize CDMS-style detectors with target masses growing from $25 \mathrm{~kg}$ to $145 \mathrm{~kg}$ and up to $1100 \mathrm{~kg}$. With a sensitivity of $\sim 1 \times 10^{-45} \mathrm{~cm}^{2}$, already the $25 \mathrm{~kg}$ phase would be complementary to LHC/ILC in the search for new physics at the weak scale.

\section{References}

[1] L. Baudis, astro-ph/0511805, in Proceedings of the XXII International Symposium on Lepton-Photon Interactions (Uppsala, Sweden, 2005).

[2] K.D. Irwin et al., Rev. Sci. Instr. 66, 5322 (1995).

[3] CDMS Collaboration, Phys.Rev. D 72, 052009 (2005).

[4] R. Bernabei et al., Phys. Lett. B 424, 195 (1998).

[5] V. Sanglard et al. (EDELWEISS Collaboration), Phys.Rev. D 71, 122002 (2005).

[6] H. Baer et al., JCAP 0309, 007 (2003), E. Baltz and P. Gondolo, Phys. Rev. D 67, 063503 (2003), A. Bottino et al., Phys.Rev. D 69, 037302 (2004), J. Ellis et al., Phys.Rev. D 71095007 (2005).

[7] D. Akerib et al. (CDMS Collaboration), astro-ph/0509259, accepted in PRL (2005).

[8] D. Akerib et al. (CDMS Collaboration), astro-ph/0509269, accepted in PRD RC (2005).

[9] R.W. Schnee et al. (SuperCDMS), astro-ph/0502435; P.L. Brink et al. (SuperCDMS), astro-ph/0503583. 